\title{
Disaster Exercise Outcomes for Professional Emergency Personnel and Citizen Volunteers
}

\author{
Ronald W. Perry*
}

It has long been argued in the research literature that conducting disaster exercises produces a variety of benefits that promote effective emergency management. In spite of nearly universal acceptance of the claim, there are few empirical studies that have explored the effects of exercises on participants. This paper reviews the role of exercises in the creation of community disaster preparedness, while making explicit the links among planning, training and exercising. Using a quasi-experimental design, the effects of disaster exercise participation on perceptions of response knowledge and teamwork are studied for police officers, fire-fighters and civilian volunteers. The exercise studied involved an annual airport disaster drill required for continuing certification. It was found that participation enhanced the perceptions of response knowledge and teamwork for all three types of participants.

\section{Introduction}

The attack on the World Trade Centres in the United States on 11 September 2001 demonstrated to the international emergency management community that terrorist incidents must be integrated into the range of threats to be actively managed. While the probability of a terrorist incident at any given time and place remains low compared to other natural and technological hazards, the World Trade Centre experience emphasised the potentially high consequence outcomes of such events. Particularly in America, there has been a significant increase in serious concern about emergency preparedness for terrorism on the part of emergency management professionals, government administrators and elected officials. The issue of promoting emergency preparedness has arisen many times in the past in connection with emerging natural and technological threats (Turner and Pidgeon, 1997; Mileti, 1999).

In virtually every instance, emergency managers have appealed to a long-held vision of creating preparedness: first plan, then train, then exercise. This sequence of activity is both time-honoured and time-tested in the area of operational applications. The value of conducting disaster exercises is emphasised in virtually every textbook on disaster planning (Rosenthal et al., 2001; Lagadec, 1997; Michaels, 1996). Disaster exercises are even institutionalised in that they are mandated by legislation and executive rules in connection with a variety of natural and technological threats in most industrialised nations (Selvarajah, 1993). In spite of almost universal respect for exercises, it remains that there is very little research precisely demonstrating their benefits (Peterson and Perry, 1998). Indeed, Borodzizc and Van Haperen (2002), echoing the concern with little research on exercises, have pointed out that crisis simulations themselves should be scrutinised and designed carefully to avoid limiting the range of 'trained experiences' to only those outcomes that are thought of and used by exercise designers. The purpose of this paper is to briefly review the conceptual bases for exercises as a component of the process of developing disaster preparedness and to report data describing selected disaster exercise outcomes for participants. In particular, the research presented here documents the differential outcomes for exercise participants who are firefighters, police officers, and citizen volunteers.

\section{Linking Disaster Planning and Disaster Management}

There has been much confusion about what constitutes disaster planning and how such activities are related to disaster response or management. One popular notion is that a disaster plan exactly specifies every action that should be
*School of Public Affairs, Arizona State University, Tempe, AZ 85282-0603. USA. E-mail: ron.perry@asu.edu 
undertaken in a disaster. This philosophy leads to the creation of lengthy plans that attempt to anticipate every possible event and prescribe correctives. Years of field research reveals that such a conception of planning is at best not constructive. In the first place, a plan should not be an all-encompassing document or tome. Plans designed like this tend to sit on shelves collecting dust and giving a false sense of security or preparedness to their writers. In the second place, it is simply not possible to anticipate every event or nuance that may arise in a disaster. Unpredictability is a defining characteristic of disasters; if we could predict every aspect in advance and devise contingencies, the event would not be a disaster (Quarantelli, 1998). Furthermore, when emergency managers attempt to implement such a plan they would find their options restricted in advance, and limits on their ability to innovate and be flexible. Thus, even planners with positive intentions would ultimately slow decision making processes, endanger response personnel and create confusion when inevitably an event occurred during response operations that was not covered in the plan.

Another view of plans is that they are preliminary preparations that anticipate action; different from disaster management which is action itself. Certainly, one should never confuse planning with management. Planning is preparing - doing the teaching, acquiring the resources - before the event. To begin planning, one identifies the major agent-generated and response-generated demands and creates strategies and tactics for fulfilling them. Management is performance. Managing requires that we use planning decision guidelines and cached resources to structure our actions, but that we assess demands as they arise and act to meet those demands in a creative and innovative fashion. As Borodzizc and van Haperen (2002: 139) demonstrate, if the problems we face are accurately anticipated in the planning process, then we are prepared and our actions will be guided effectively by the elements of the plan. If not, we must assess the environmental contingencies we face and devise a workable response using plan-based resources and guidelines, but supplementing them with improvised resources and decision rules.

Thus, while planning is not management, effective planning should be very closely related to management. They supplement and compliment one another and should not be thought of as separate. It is possible to conceive of disaster management or response as a series of decisions made by emergency managers. Viewed this way, planning provides the framework for emergency response decision-making and structures the options from which a decision-maker can choose to address practical challenges. Planning can be thought of as the decision preparations that the emergency manager makes during 'normal' times that help to guide actions during disasters.

\section{The Disaster Plan and Exercises}

The disaster plan is the written document or map for disaster management generated by any given political jurisdiction or private organisation (Lindell and Perry, 1992). Written plans may vary widely in scope, detail, structure, purpose and elaborateness. In every case, the disaster plan is the product of the planning process, thereby becoming the principal connection between the disaster planning activity and the disaster response. The construction of the plan is such that it addresses issues that are relevant to response. The focus is upon writing down a description and analysis of needs that arise and actions that can be undertaken and resources that should be assembled to support the actions. Put another way, the planning process anticipates demands and devises strategies and tactics, linked with resources, for meeting them. The product of an effective planning process is reflected in jurisdictional disaster preparedness. Another important connection between plans and action is achieved by conducting exercises. In Canada, Australia and the United States, exercises are sometimes referred to as "drills" and in Europe and Great Britain they are sometimes called "simulations." Such activities are usually mandated in a comprehensive emergency plan, but may also be seen as part of the planning - training - exercising process. Thus, through the process of planning, one documents the knowledge, skills and abilities that are likely to be needed during a given disaster response. Once this information is identified, emergency managers can design training that imparts the requisite knowledge, skills and abilities. Then one can plan exercises that involve simulated incidents and test not just overall response capability, but the extent to which participating personnel have mastered the training that they have undergone (Rosenthal and Pijnenburg, 1991).

From the jurisdictional standpoint, disaster exercises serve a variety of functions. One of these is the critical function of detecting difficulties in executing the strategy or tactics proposed in the plan. Since an exercise is an operational test, operational failures or weaknesses are identified. Problems that might be revealed in an exercise can be specific and procedural, as well as more general. For example, during an exercise, one might learn that hazardous materials technicians in full protective gear are unable to clearly see a particular instrument, or equally important, it might be discovered that an interagency agreement to share ambulances was not viable. 
Another function of exercises is that they allow personnel from responder groups and agencies to develop working relationships with one another. The smoothness and effectiveness of inter- and intra-organisational relationships promotes successful performance during the response phase. Exercises offer organisational personnel the opportunity to develop a history of interaction and co-operation that enhances their ability to work together when disaster strikes.

Exercises also constitute a type of public information. At one level, publicity associated with an exercise 'informs' the public that government is aware of environmental threats, has a plan for dealing with threats, and is actively engaged in testing those plans. This type of 'public information' function appears to be particularly useful in the context of terrorist threats. In this case, while the dangers of terrorism are known to the public, the specific mechanisms of destruction -incendiary explosives, radiation, chemical agents and biological agents - are unfamiliar to the public, difficult or impossible to detect with human senses alone, capable of causing immediate death, and also capable of creating negative consequences that may be delayed for some time (Lindell and Perry, 2003). All of these features are intensely fear producing (Slovic et al., 1980). While exercises do not change levels of public knowledge of all agents and consequences, the agent used in the publicised exercise becomes more 'real' to citizens and emergency managers are witnessed in the response process. Such observations permit members of the public to begin to attribute 'credibility' to emergency management efforts, which in turn increases the probability that citizens will comply promptly with recommended emergency measures in a time of disaster (of any type). In connection with this public information function, exercises also give emergency managers practice dealing with the press (or mass media in general), and permit refinement of the role of the unit public information officer. At the same time, exercises help members of the press to understand the conduct of emergency operations and reduce the chance that an adversarial relationship will develop between media representatives and emergency managers.

Finally, in addition to testing training programs, exercises serve an educational function in themselves. New responder personnel have an opportunity for "hands on" exposure to emergency equipment, vehicles, procedures and protocols. There is also an educational function for elected and appointed public officials, particularly those who are not directly involved in emergency planning and response. The conduct of an exercise informs such officials of emergency management efforts, and offers an opportunity for them to 'see' the performance of various agencies.
Disaster Preparedness: Planning, Training, Exercising

Both emergency managers and researchers have devoted much time to understanding and defining disaster preparedness (Gillespie and Colignon, 1993; 't Hart, 1997). While it is not possible in this forum to exhaustively deal with the myriad aspects of preparedness, it is possible to provide an overview of the relationships among three critical preparedness components: planning, training and exercising. In this case, concern lies with planning in the narrowest sense of that term. For this discussion it is assumed that the broader emergency functions such as vulnerability analysis, technical analysis of structural and non-structural options, and political and technical feasibility reviews, have been completed. It is assumed here that emergency officials have identified a specific threat agent and an accompanying constellation of measures (representing the state of knowledge and technology) to be undertaken in response to the threat. In this context, planning involves the construction and codification of strategy and tactics into an executable series of actions or tasks. Plans address all aspects of the response, including personnel, equipment, contingency issues, policy issues, and inter-organisational and inter-governmental relations. The plan represents, then, a blueprint for addressing all aspects of a particular environmental threat.

Once a plan is created, its implementation revolves around the logistics and protocol needed to execute the specified series of tasks. Implementation usually begins with a detailed assessment of capacity. Agencies who provide different functions under the plan must be evaluated for their ability to comply. Personnel compliments and equipment need to be brought to plan specifications. In connection with or as a product of this assessment, a variety of training needs may be identified. These include both training and education of personnel regarding the threat, the response processes and procedures, and the use of the equipment called for under the plan. In this context, training is the activity that translates information defined as needed by the plan into a coherent program that can be imparted to responders.

Broadly speaking, exercises represent constructed opportunities to test the protocols and equipment specified under a plan and taught in the training phase. Exercises may be seen as a form of training in the sense that individuals are rehearsing response measures. Ultimately, however, exercises provide the forum or context to test the effectiveness of both the training program and the plan, as well as the ability of personnel to execute the plan.

The creation of meaningful disaster exercises demands that the event test personnel, protocol 
and equipment (Emergency Management Institute, 1990; Home Office, 1998). Thus, developing an exercise requires the elaboration of milestones designed to simulate an event or incident that approximates the threat addressed in the planning and training phases (Hermann, 1997). A critical initial exercise milestone is to set the objectives for the exercise. These may be broad or narrow, depending on the scope of testing that is desired. For example, in exercising a biological hazard plan for a smallpox agent, one might choose to devise an exercise built solely around testing the warning phase activity. Such an exercise scenario might involve tasks associated with evaluation of medical surveillance data by local emergency managers, making a decision to evacuate or quarantine private homes and businesses, constructing a warning message and notification and mobilisation of the agencies involved in disseminating the message to the public. Once testing or exercise objectives have been set, one must devise an event scenario. Such a scenario must posit an event or series of events that offer an opportunity for plan specifications to be implemented. In some exercises, the creation of the scenario is particularly intricate involving not only simulation of an event, but also victims and physical damage. The management of an exercise is somewhat similar to a major stage production in that there must be realistic actors and props, with detailed stage direction (training). Often the utility of the exercise depends in part upon the extent to which those participating in the exercise find the activity believable or compatible with their knowledge of potential events. Exercises are usually generated with specific goals and objectives that are carefully related to the vulnerability pattern and planning activity of a given jurisdiction.

When considering exercises as part of community preparedness, it is acknowledged that the detail and extensiveness (scope) of an exercise represent different levels of testing of community-wide capabilities. Trainers and emergency managers usually identify three general types of exercises (Daines, 1991). These types are labelled tabletop, functional, and full-scale exercises. A tabletop exercise is the least complex of all exercises, and focuses upon a primarily verbal recounting of an incident. Participants in these types of exercises are usually gathered in a single location, sometimes in the same conference room. Typically these exercises begin with a simulated event (disaster) narrative after participants have been assigned roles in the emergency response system being exercised. Often participants verbally respond to event demands by describing the actions they would initiate, since contacts with other responders or agencies are normally simulated. Exercise managers (usually called controllers) implement the exercise proto- col and monitor the responses of participants, sometimes injecting event variations into the exercise process to test specific exercise objectives. In these types of exercise, evaluation and self-critique may be conducted after the exercise is completed or progressively through the event. Tabletop exercises are the least formal type of exercise and tend to achieve very generic assessments, but are very cost-effective and particularly useful when new protocols are being introduced into existing response systems or when previously unmanaged threats have been identified. At best, however, tabletop exercises do not achieve the realism of simulated execution in the field - the actions tested are actually 'action intentions', not operational executions.

Compared to the tabletop experience, a functional exercise represents greater complexity in testing planning and training. In keeping with their name, these exercises select one or a small number of functions under a disaster response plan as a focus for exercise. For example, in the context of a plan to respond to a terrorist generated explosion that dispersed radioactive material (called a 'dirty bomb'), one might conduct a functional exercise to test the Emergency Medical Services (EMS) segment of a response plan. The functions tested might be victim decontamination, triage, scene treatment, and transportation to hospital for extended treatment. Similarly, a functional exercise could be focused on the Law Enforcement segment using the same scenario but addressing objectives related to scene isolation and control, evidence gathering, and perpetrator identification. Consequently, depending upon the nature of the plan tested, a functional exercise may involve a single responder agency or many. Usually, functional exercises are conducted in real time, in the field, with operational personnel executing their functions using appropriate equipment. This normally demands that the exercise staff include actors, such as simulated victims in an EMS exercise, and that the scenario be supported with appropriate props consistent with the threat. Unlike the tabletop exercise, realism is important in functional exercises.

Finally, the most complex form of exercise is the full-scale exercise. The purpose of the full-scale exercise is to test all or a major portion of the functions specified in an emergency response plan. To accomplish tests of multiple functions by definition requires the statement of many exercise goals (objectives) and sub-goals, the participation of multiple responder agencies, and the requirement for a high level of realism in the scenario. As a result, full-scale exercises are major enterprises that demand many resources, a full staff of evaluators and controllers, a compliment of actors (victims and other event-impacted personnel), and realistic simulations of the physical damage and other consequences of the 
event. Participants at all levels must literally execute their tasks under the disaster plan on the operational field in real time.

\section{Expected Benefits of Disaster Exercises for Participants}

The generic benefits of disaster exercises, described above, included benefits to the jurisdiction, testing of the plan and equipment, and logic of response. From the standpoint of emergency management, exercises provide an opportunity for participants to demonstrate their knowledge, skills and ability. In addition to the demonstration of trained ability, exercises impinge directly on the individual responders. That is, there are social psychological effects of exercise participation that are associated with the more concrete activities executed in the exercise. The emphasis in the research literature is often on exercises as an opportunity for planners, trainers and managers to observe and rate the performance of responders. But the experience of the exercise also shapes the responders' perceptions of the emergency management process and the actors involved in the response. These judgements on the part of responders can seriously impact the larger emergency management system. Jurisdictional emergency managers, planners, and trainers must be seen as credible professionals in the eyes of responders to achieve any level of community protection (Drabek, 1987, 1990). Similarly, to effectively execute their jobs, emergency responders must perceive that their training has been adequate and that their equipment is appropriate to their assigned tasks. Each time emergency responders participate in an exercise their opinions and perceptions of these and a host of other related issues are shaped.

Peterson and Perry (1999) have reported that exercise participants experience changes in perceptions of teamwork, efficacy of response networks, adequacy of training, adequacy of equipment and the levels of risk associated with emergency responder jobs. These researchers cautioned readers that their data contained a potentially serious limitation: only hazardous materials technicians from a fire department were participants. There has been speculation in the literature that professional emergency responders may experience different benefits from exercises than citizens, and even emergency responders from different disciplines may have different experiences. It is important to understand differences in response patterns as an avenue to focusing and improving trainee reception to planned exercises. In addition to the concern with differential impacts, Peterson and Perry (1999) have also suggested that further experimental research is needed on social psy- chological benefits of exercising, specifically upon responder impressions of the ability and competence of co-workers. The present research will attempt to address these two issues. The idea that participating in an exercise has differential impacts on responders from different response disciplines will be examined by including police, fire-fighters and civilians among research comparison groups. The question of participation impacts on responder perceptions of coworkers will be addressed by measuring perceptions of teamwork and knowledge of incident management before and after the exercise experience.

Although sparse, the available research literature was useful in choosing particular responder perceptions for study. Both training manuals and anecdotal evidence emphasise that responders involved in exercises are able to observe first hand levels of teamwork in the process of managing a threat (Shapiro, 1995). Teamwork is seen in this context as the belief that individual responders can successfully engage with fellow responders to achieve a common goal. In particular, Kartez (1988) argues that since exercises demand teamwork across agencies, a successful outcome should increase participant perceptions that teamwork can be achieved. Similarly, the United States National Response Team (1990) manual on exercising identifies team building as a major consequence of exercise participation.

Peterson and Perry (1999) used global measures to test this assertion among fire services professionals and found that (in the case of a successful exercise) participants did perceive that levels of teamwork increased as a function of exercise participation. While global measures are useful, it is also important to document exactly which aspects of teamwork ability are perceived to change. While there is no specific literature to use as a guide, teamwork can be seen as having at least two referents. The first is the extent to which exercise participants are perceived to simply be able to co-ordinate their actions to execute specific response protocols (Hildreth, 1989). Furthermore, co-ordinated actions or team ability may be seen in terms of respondent colleagues from the same emergency discipline, and respondent colleagues from different emergency disciplines. Thus, one would examine fire-fighter perceptions of team skills among fire-fighters and fire-fighter perceptions of the ability of fire and police personnel to achieve co-ordinated action. The second referent may be captured as the exercise participant's knowledge of the technical aspects of the job of fellow participants who come from other emergency disciplines. Even within the same discipline, one would not expect a respondent to have detailed knowledge of other participant specialities. Thus, a fire department hazardous material's technician would not be 
required to have technical knowledge of a paramedic's job in order to effectively respond with the paramedic. On the other hand, the basis of emergency response for all disciplines rests with the concept and implementation of incident management systems (Brunacini, 2002). Consequently, it can be argued that to achieve optimum incident response (certainly within exercise scenarios) members of different response disciplines should understand at least the rudiments of the incident management systems used by all participating disciplines or agencies.

\section{Research Design and Hypotheses}

The aim of the present study, using a quasiexperimental research design, is to examine the teamwork perceptions of police officers, fire fighters and trained civilians who participated in a large-scale municipal disaster exercise. The event studied here was an airport emergency response exercise that involved the participation of multiple municipal departments with a federal organisation. To obtain access to study the event, the researchers promised that the name of the municipality and of organisations directly participating in the exercise would not be revealed in reports or published documents. An exercise scenario was developed that satisfied the external exercise requirements imposed on each participating agency. The perceptions of members of each group were measured before and after exercise participation (Campbell and Stanley, 1964). The before measure or pretest was made as part of standard exercise scenario briefing approximately seven days before the exercise took place. The after measure (or posttest) was a part of participant debriefing sessions on the closing day of the exercise. Both the pretest and posttest measures were obtained as part of a one page self-administered questionnaire. This research design enables us to examine initial levels of each impact perception for each group and to record the levels of the same perceptions after the exercise. The before-after change score (called a gain score) indicates how much change occurred for each perceptual dimension. The exercise participants studied were 40 fire-fighters, 40 police officers and 20 Emergency Operations Centre (EOC) volunteers.

Assuming that the exercise is successful, one can devise three hypotheses regarding the impacts of participating in a disaster exercise. First, exercise participation should increase participant's beliefs that they understand the incident management systems used by participants from different disciplines. Second, exercise participation should increase the perception that like discipline responders can work together as a team. Third, exercise participation should in- crease the perception that responders from one discipline can work together as a team with responders from other disciplines.

It is important to emphasise that the hypotheses suggest only that exercise participation affects these three perceptual dimensions. The direction of the effect, in terms of our substantive understanding, would be a function of the extent to which the participants perceived the exercise outcomes to be successful. In an unsuccessful exercise, one would expect that participant confidence levels in each dimension would decline. On the other hand, a successfully executed exercise would logically tend to increase perceptions of knowledge and teamwork.

Four variables, representing the areas of teamwork perception identified above, were measured using single Likert-type scale statements. The operational definition used for the measure of incident management system knowledge was: "I am confident that I understand the elements of police incident management systems." The statement as written was given to fire personnel. When measuring police perception of fire department incident management systems, the same statement was used except that 'fire department' was substituted for 'police'. No measure of understanding police and fire IMS was included for the civilian emergency operating centre volunteers, since these systems were not a part of their experience. Three statements were used to assess specific perceptions of levels of teamwork. The first addressed teamwork among individuals from the same discipline or performing the same function in the EOC. The general Likert item was: "I am confident that fire-fighters assigned to an incident can work effectively as a team." For police, the same statement was used with the substitution of 'police officers' for 'fire-fighters'. For EOC volunteers, the statement was slightly amended to better fit their assignment "I am confident that the people assigned to my EOC section can work effectively as a team". The second statement addressed perception of teamwork among individuals in other responding disciplines: "I am confident in the ability of the fire department team to work effectively together in an incident." For fire-fighters, the term "police" was substituted for "fire". For the EOC volunteers, the statement was "I am confident that the people assigned to the other EOC sections can work together to achieve their section assignments". Finally, the last variable examined participant perceptions that members of different disciplines can work in concert. The statement for professional emergency responders was "I am confident that police and fire response teams can effectively work together." For EOC volunteers, the statement was "I am confident that all the volunteers assigned to the four EOC functions can effectively work together." 
Thus each variable was measured as the exercise participants agreement with a simple, declarative statement regarding the reference perception. An expanded Likert-type response format was developed and used for each statement (Edwards, 1957). The response format was composed of 7 assessments of the extent to which the individual agreed with the content of the Likert item: Strongly Agree, Agree, Somewhat Agree, Neither Agree nor Disagree, Somewhat Disagree, Disagree, Strongly Disagree. Thus, emergency responder ratings for each perceptual area varied from 1 through 7 . Reverse coding was used so that the value 7 was assigned to "Strongly Agree" and 1 to "Strongly Disagree". Therefore, smaller numbers represent lower levels of each variable (knowledge and teamwork).

\section{Exercise Overview}

The exercise described here was used to test a portion of the airport disaster plan maintained by a large municipality in the western United States. The functional exercise was designed in connection with a governmental requirement for an annual jurisdictional exercise. The exercise required eight and one-half hours to complete. In brief, the scenario for the exercise focused upon the arrival at the regional airport of a commercial aircraft (a Boeing 737), with 120 passengers aboard. Five minutes prior to landing, passengers and crew noticed a vapour cloud in the passenger compartment. All passengers began coughing, tearing, and reported trouble breathing. The crew was unable to immediately identify a source for the cloud. The aircraft commander ordered oxygen used by passengers and crew, began venting the aircraft, and initiated emergency landing procedures for the airport. Once on the ground, the aircraft taxied to the area designated in the emergency plan for arriving, contaminated aircraft with ill passengers.

The disaster plan notification sequence brought police, fire fighters and the airport and municipal emergency managers to the scene. In this case, the scene was composed of the aircraft with simulated smoke inside, passengers and crew still onboard, with 5 passengers simulating lost consciousness and an additional 10 passengers were moulaged (that is, wore injury-simulating makeup) to present various non-fatal trauma injuries induced by a difficult landing. The scenario specified that emergency responders would view the scene and then activate the municipal emergency operations centre (EOC), located offsite from the airport.

The exercise participants of interest in this research included professionals from two emergency disciplines, police and fire services, and citizens. The fire-fighters served in two roles: some were paramedics and others were members of the hazardous materials response team. Police officers participating also came from two units: members of the bomb squad and those who were part of a specially trained neighbourhood response team. The city employees (citizen volunteers) who were trained and supported the efforts of senior advisors and resource managers in the emergency operations centre were also studied. Both the police officers and fire-fighters were professionally certified for their jobs, had held their response positions for more than one year, had participated in other exercises in the past, and were specifically trained in the elements of the response plan. The citizens studied did not constitute a professionally certified force. Instead their mission was generic, to receive, record and relay information, using the equipment in the emergency operations centre as a means of supporting the activity of the EOC Commander and staff. Information was received from commanders at the scene, from airport authorities, from local government officials, and citizens, and potentially relayed to all of these sources as well as to the EOC commander and staff. The volunteers did not serve as emergency dispatchers for the deployment of police, fire or other emergency management personnel or resources. The city employee volunteers had been given an initial eight-hour training session for EOC support staff. Each volunteer receives 4 hours of annual EOC familiarisation and refresher training. None of them were employed in the police or fire departments. All of the civilian volunteers had completed some university education, all had participated in at least one previous incident and one previous exercise in the EOC.

The emergency response tasks rated were different for different classes of participants. The fire services hazardous materials commander was required to do continuing incident assessment, establish scene command, and order that the EOC be activated. The hazardous materials team accomplished initial entry, supervised evacuation of ambulatory victims, extracted nonambulatory victims, performed product sampling, identification and abatement. The police bomb squad was tasked with determining no secondary devises (designed to injure first responders) were present, and backing up the hazardous materials team. The special police unit established perimeter, maintained security, took possession of evidence and initiated investigation. The paramedic fire fighters were charged with decontamination of victims, decontamination of entry teams, conducting triage for victims, initiating treatment and transporting victims to hospital care. All of these functions were pursued at the scene. At the emergency operations centre, the expectations for the volunteers were more generic. The principal requirement was that each volunteer attend to an assigned support post (as 
part of the citizen information telephone line, the operations support group, the administrative support group, the incident planning support group or the logistics support group). While at the post, the volunteers recorded information, answered questions, relayed information, used a computer-based emergency management software program, and used other administrative equipment.

\section{Results}

The results of the present study provide empirical evidence that disaster exercises produce changes in the perceptions of participants regarding knowledge of response systems and team capability. Furthermore, the impacts detected were observed for three categories of individual. The first category was "professional emergency responder," represented by fire-fighters and police officers. While the exercise impacts for these two classes of professional responder were similar, it is interesting that the effect magnitudes are slightly different for each group. The third category of participant was civilian volunteer. It has long been speculated that non-professional emergency response volunteers may experience relatively greater benefits from exercise participation. The data presented below indicate that differential benefits are more a matter of degree than kind when it comes to perceptions of teamwork.

Understanding the Incident Management System. Because the systems are designed to address fundamentally different types of event, one can expect that the details of incident management systems used in law enforcement and fire services will differ from one another. At an operational level, it is only important that each category of actors have sufficient familiarity with the other system to be able to execute tasks without interfering with operations flow. There is much anecdotal evidence that police have little appreciation of fire incident management systems and that police incident command systems are a mystery to fire-fighters. Yet to effectively collaborate at the same scene, some degree of familiarity must exist for each group.

\section{STATISTICAL DATA FOR IMS UNDERSTANDING}

The basis of experimental designs rests upon comparisons of subjects before and after exposure to a 'treatment' or intervention. In this experiment, the intervention was the experience of participating in the disaster drill. Three groups were exposed to the drill: volunteer civilian personnel, police personnel and fire fighters. Our interest in change focused upon two categories of perceptions: perceptions of understanding of incident management systems and perceptions of teamwork. Each of these categories of perception was measured before and after the groups participated in the drill. The statistical system used to examine comparisons from an experimental design is the Analysis of Variance, which offers systematic comparisons of mean group scores at the 'before' and 'after' measurements. The technical data from these analyses is summarised below.

At the pretest, the mean fire-fighter rating for understanding police IMS was 2.8 (standard deviation $=.91$ ), while the police mean rating for understanding fire IMS was 2.9 (standard deviation =1.08). The post test mean for firefighters was 4.60 (standard deviation $=.74$ ) and for police was 3.77 (standard deviation $=.77$ ). Thus, the average gain score (post test minus pretest) for fire-fighters was +1.8 units (standard deviation =1.01) on the scale and police officers increased by .87 units (standard deviation $=1.04$ ). While each gain score represents a substantively meaningful increase in perception of knowledge of IMS, simple analysis of variance (ANOVA) indicates that the increase for firefighters is statistically significantly greater than that for police officers $\left(F_{1,78}=16.12, p<.001\right)$.

Fire-fighters were asked to rate the extent to which they agreed with the statement that they understood police incident management systems and police were asked about fire department incident management. At the pretest, the fire-fighters indicated that their understanding of police IMS was low. Similarly, police perceived that their understanding of fire IMS was also low. Hence, the average score for both fire fighters and police showed that they would "somewhat disagree" with the claim that they were confident in their understanding of police or fire IMS. When asked the same question after the exercise, the mean scores of fire and police personnel substantially increased. It should be pointed out that the amount of increase for fire fighters was greater than the increase for police. Thus, after the experience of the exercise fire personnel felt more confident in their understanding of police IMS than police felt with fire IMS. Without regard to magnitude, however, it is critical to note that exercise participation was paired with high levels of confidence among both police and fire fighters. 


\section{Measures of Teamwork}

Three aspects of perception of teamwork were examined and are presented serially here. The first measure addresses the participant's perception that the responders from their own discipline can work together effectively as a team. Since this is a rating of one's own colleagues - with whom training and daily work were undertaken - it was expected that the initial ratings would be high and that the exercise experience would cause them to change very little. At the pretest phase, fire-fighters rated their colleagues higher than the other groups; indeed the fire fighters rating was close to the upper limit of the scale. The police officers were just slightly lower than the average fire-fighter ratings, but still showed a very high level of confidence that police can work as a team. One possible explanation for the difference is that the fire-fighters participating in this exercise came from two fire disciplines that often work together, while the police were from more diverse police disciplines that work slightly less frequently on the same scene. The difference between fire-fighters and police is interesting, but not large enough to demand substantive attention. The EOC volunteers showed the lowest level of confidence that other volunteers assigned to their response section could work as a team. Thus, at least at the beginning of the exercise, EOC volunteers on average did not make a concrete commitment regarding the confidence in which they held their fellow responders.

While each of the three groups increased their mean confidence scores following the exercise, the size of the increase for professional responders was low, while the magnitude for civilian volunteers was very high. The average increase in confidence among fire-fighters was smallest, indicating that participation in the exercise increased collegial confidence only slightly. It should be pointed out that the initial fire fighter rating was so high, that we are probably experiencing a ceiling effect. Similarly, police officers average increase in confidence was only slightly higher than fire fighters. Thus, both police and fire personnel began with very high levels of confidence in colleagues and still increased slightly as a function of participating in the exercise. EOC volunteers began much lower on the scale and increased substantially-about twice as much as the increase among police and fire personnel. Consequently, while exercise participation increased confidence for all three groups, volunteer personnel clearly benefited most with respect to this dimension.

The second teamwork question addressed the extent to which one had confidence in other responders from a different professional discipline, or for the EOC volunteers, their level of confidence in other volunteers working in differ- ent functional areas of the EOC. At the pretest measure, both fire and police personnel were cautious about estimating confidence that should be placed in each other's ability to respond as a team. The mean score for fire-fighters rating police teamwork prior to the exercise was at the middle of the measurement scale. Similarly, police rating of fire-fighter teamwork was at the centre of the measurement scale. Interestingly the police had slightly higher confidence

\section{STATISTICAL ANALYSES FOR TEAMWORK}

With respect to teamwork, each of the groups evaluated the extent they felt their own group could work as a team. At the pretest phase, firefighters rated their colleagues high on teamwork, with a mean of 6.17 (standard deviation $=.38$ ). The police officers were just slightly lower than the average fire-fighter ratings at a mean of 5.42 (standard deviation $=.54$ ). The EOC volunteers showed the lowest level of confidence that other volunteers assigned to their response section could work as a team at a mean score of 4.55 (standard deviation $=.80$ ). After the exercise each group increased their confidence in teamwork within their group. The average increase in confidence among fire-fighters was lowest at .45 (standard deviation $=.50$ ). Police officers average increase in confidence was .57 (standard deviation $=.59$ ). EOC volunteers increased an average of 1.80 scale units (standard deviation =.89). An ANOVA conducted for the three groups by average gain score reinforces that there are statistically significant differences between the professional responders and the volunteers $\left(F_{2.98}=33.47, p<.001\right)$.

The second teamwork question addressed the extent to which groups had confidence in other responders from a different professional discipline, or for the EOC volunteers, the level of confidence in other volunteers working in different functional areas of the EOC. The mean pretest score of firefighters rating police teamwork was 3.58 (standard deviation $=.56$ ) and of police rating firefighter teamwork, 3.97. After the exercise, these assessments increased substantially. The average increase in fire-fighter assessment of police teamwork was 1.27 scale units (standard deviation $=.90$ ). The police officer perception of firefighter teamwork ability rose 1.75 scale units (standard deviation $=.49$ ). At the pretest, the EOC volunteers rated other volunteers from different EOC functions cautiously: the mean rating was 4.25 (standard deviation=.44). After the exercise, the EOC volunteers showed a very large increase in confidence, an average of 1.9 scale units (standard deviation .55). A simple analysis of variance of the gain scores confirms that the 
magnitude of increase was greatest for volunteer workers, and less for professional emergency managers, and that these differences were statistically significant $\left(F_{2.98}=7.09, p<.001\right)$.

The last teamwork issue addressed measured participant confidence that incident responder's could effectively work together as a team. The average initial ratings that police and fire personnel could effectively respond together was 3.73 (standard deviation $=.46$ ) for fire-fighters and 3.95 (standard deviation $=.45$ ) for police officers. EOC volunteers showed an average rating of 4.15 (standard deviation $=.57$ ). After the exercise, Fire-fighter's showed an average increase of 2.25 scale units (standard deviation $=.54$ ) in confidence, police officer confidence increased on average 2.17 scale units (standard deviation $=.59$ ) and EOC volunteers confidence levels increased an average of 1.90 scale units (standard deviation $=.64$ ). All three groups showed large (and similar) increases in the magnitude of confidence (there were no statistically significant differences between gain scores, $\left.F_{2.98}=2.50, p>.05\right)$.

in fire-fighter team efforts than fire-fighters had in police. Since the fire fighters and police officers studied here were not command officers, their experience with each others incident performance was limited, making such midrange judgements prudent or conservative assessments of levels of teamwork. After the exercise, these assessments increased substantially. The average increase in fire-fighter assessment of police teamwork was again slightly lower than the police officer perception of fire-fighter teamwork ability. The differential between the two levels of increase may be a function of differing visibility of the police and fire personnel. The functions executed by police officers (and the timing of the functions) were such that through most of the exercise, fire-fighter activity was visible to most police officers. On the other hand, most firefighters were assigned to one of three sectorsvictim decontamination, victim treatment or victim transportation to hospital - where the activity of police was largely invisible. Indeed, the only fire-fighters who directly observed police operations were the hazardous materials technicians who worked near the police bomb squad. Thus, it is possible that fire-fighters rated police teamwork slightly lower because they simply did not witness police performance. At the pretest, the EOC volunteers cautiously rated other volunteers from different EOC functions slightly above the mid-point of the scale. After the exercise, the EOC volunteers showed a very large increase in confidence. Each group of participants then, after engaging in the exercise, increased their levels of confidence in the teamwork ability of the participants from other emergency response disciplines or training functions. And the statistical analysis confirms that the magnitude of increase was greatest for volunteer workers, and less for professional emergency managers, and that these differences were statistically significant.

Finally, the last teamwork issue addressed focused upon participants confidence that incident responder's could effectively work together as a team. All three groups were cautious regarding this assessment at the pretest time. The initial ratings that police and fire personnel could effectively respond together were at the middle of the confidence scale for both fire fighters and police officers. EOC volunteers showed a slightly higher confidence before the exercise than the professional groups. Although there are small numeric differences between the assessments of the three groups, each group expressed a neutral assessment. Thus, all three groups began the exercise expressing neither confidence nor a lack of confidence in overall teamwork ability. Interestingly, the largest impact of exercise participation on the three teamwork measures is found at this level of perceived confidence that members from the different disciplines can effectively work together. Fire-fighters showed the largest increase followed by police and EOC volunteers. In spite of the differences in magnitude, each group increase constituted a near doubling of their initial score. These results underscore that exercise participation has a tremendous impact on perceptions of responder teamwork ability.

\section{Perceptions of Single Most Important Experience}

There has been much discussion, but little empirical investigation, regarding what exercise participants believe that they gain from exercises (Peterson and Perry, 1999). Although the information is subjective, participants in this study were asked the open-ended question "what was the single most important benefit you obtained by participating in this exercise." The responses to this question by professional emergency responders were substantively different than those given by the EOC volunteers. Among the 80 firefighters and police officers, 53 answered this question and 27 did not. All 20 of the EOC volunteers answered the question. Among the professional responders, 31 felt that the single most important benefit as the "opportunity to see the unified (police-fire) command system in action". The next most commonly mentioned benefit in frequency of mention was the opportunity to make "cross department" contacts with fellow responders (cited by 10 professionals). Eight of the professional responders (all either members of the fire department hazardous materials teams or the police bomb squad) viewed the "opportu- 
nity to work in level A protective garb" as the principal benefit of participation. Finally, four professionals cited the discovery of minor difficulties in response protocol as a most important benefit. Thus, the professional emergency responders tended to see the value of the exercise in terms of experiencing unified command, making contacts across department lines, and work practice. The citizen volunteers in the EOC tended to cite either work practice or the acquisition of new skills as principal benefits of exercise participation. Thus, eight volunteers felt that the value of the exercise lay in opportunities to learn; they cited specifically learning how to operate new machines, new computer software, obtaining information about the support role of the EOC, and learning about the response protocols of the police and fire departments. Seven of the volunteers reported that the principal benefit of the exercise was in improving their ability to execute tasks that they had been trained to accomplish. Half of these expressed the opinion that their training was "book based" and the exercise allowed them to see if they could "do the job for real". Three of the volunteers reported that the value in exercise participation was the opportunity to practice their assignments and two others felt the primary value of the exercise was that it "allowed observation of an incident in progress".

\section{Conclusions}

The experimental data document several important conclusions about exercise participation with regard to its impacts on perceptions of the response and of levels of teamwork. With regard to knowledge of incident management systems, participating in an exercise increases perceived knowledge among professional and volunteer personnel. In the data presented here, both police and fire personnel believed that they learned much about the others incident management, although the magnitude of effect was greater for fire-fighters than for police officers. With respect to teamwork, three specific dimensions of the concept were tested. With respect to police and fire-fighters, both groups began the exercise with very high levels of confidence in the ability of their own departmental colleagues to perform as a team. After the exercise, these perceptions of confidence increased, but were so high initially, that the increases approached the upper limit of the measurement scale even though they were small. Among civilian EOC volunteers, the initial confidence levels were much lower, but the magnitude of increase was much higher.

When asked about confidence that responders from other areas and disciplines would be able to work as a team, all three groups were cautious before the exercise, but each group increased significantly in confidence following the exercise. The increase in confidence was smallest for firefighters, somewhat larger for police, and greatest for civilian volunteers. The exercise had the greatest impact on participant perceptions that all of the responders could work effectively together. Again, prior to the exercise, members of all three groups rated confidence in collective teamwork near the scale midpoint. After the exercise, levels of confidence among all three groups rose by nearly double, approaching the upper limit of the measuring scale.

In terms of statistical measures before and after, participating in a disaster exercise resulted in increases in perceptions of incident management protocol and teamwork for fire-fighters, police officers and civilian volunteers. The differences that arose in these cases were principally differences in magnitude of increase. That is, civilian volunteers increased more than the two professional groups in perceptions of within group teamwork ability and in their perceptions of the teamwork ability of responders from other disciplines. Professional participants showed a greater increase than volunteers in the perception that the exercise stimulated higher levels of co-operative teamwork among responders from different disciplines. More subjectively, when defining the value of the exercise experience, professional responders tended to cite opportunities to observe joint command and to make cross-departmental contacts, while volunteers stressed the acquisition of new knowledge and reinforcement of training.

\section{References}

Borodzicz, E. and van Haperen, K. (2002), 'Individual and Group Learning in Crisis Simulations', Journal of Contingencies and Crisis Management, 10, (3), 139148.

Brunacini, A.V. (2002), Fire Command: The Essentials of IMS, National Fire Protection Association, Quincy, Massachusetts.

Campbell, D. and Stanley, J. (1964), Experimental and Quasi-Experimental Designs for Research, Rand McNally, Chicago.

Daines, G.E. (1991), 'Planning, Training and Exercising', in Drabek, T.E. and Hoetmer, G. (Eds.), Emergency Management, International City/County Management Association, Washington, D.C., pp. 161-200.

Drabek, T.E. (1987), The Professional Emergency Manager, University of Colorado Institute of Behavioral Science, Boulder, Colorado.

Drabek, T.E. (1990), Emergency Management, SpringerVerlag, New York.

Edwards, A. (1957), Techniques of Attitude Scale Construction, Appleton-Century-Crofts, New York.

Emergency Management Institute (1990), Exercising Emergency Plans under Title III, U.S. Government Printing Office, Washington, D.C.

Gillespie, D.F. and Colignon, R. (1993), 'A Structural Change in Disaster Preparedness Networks', Inter- 
national Journal of Mass Emergencies and Disasters, $11,(2), 143-162$.

Hermann, C. (1997), 'Conclusion, The Multiple Payoffs of Crisis Simulation', Journal of Contingencies and Crisis Management, 5, (4), 198-206.

Hildreth, R. (1989), 'Portrait of a Disaster', American City and County, 104, (12); 40-41.

Home Office (1998), The Exercise Planner's Guide, HMSO, London.

Kartez, J.D. (1988), Emergency Planning Baseline Data Report, International City/County Management Association, Washington, D.C.

Lagadec, P. (1997), 'Learning Processes for Crisis Management in Complex Organizations', Journal of contingencies and Crisis Management, 5, (1), 24-31.

Lindell, M.K. and Perry, R.W. (1992), Behavioral Foundations of Community Emergency Planning, Hemisphere Books, Washington, D.C.

Lindell, M.K. and Perry, R.W. (2003), Risk Communication in Multi-Ethnic Communities, Sage Publications, Thousand Oaks, California.

Michaels, J.V. (1996), Technical Risk Management, Prentice Hall, Upper Saddle River, New Jersey.

Mileti, D.S. (1999), Disasters by Design, John Henry Press, Washington, D.C.

Peterson, D.M. and Perry, R.W. (1999), 'The Impacts of Disaster Exercises upon Participants', International
Journal of Disaster Prevention and Management, 8, (4), 241-254.

Quarantelli, E.L. (1998), Defining Disasters, Routledge, London.

Rosenthal, U., Boin, A. and Comfort, L. (2001), Managing Crises, Charles Thomas, Springfield, Illinois.

Rosenthal, U. and Pijnenburg, B. (1991), Crisis Management and Decision Making: Simulation Oriented Scenarios, Kluwer Academic Publishers, Boston.

Selvarajah, C.T. (1993), 'Training System Effectiveness in Australia', Human Systems Management, 13, (3), 295-302.

Shapiro, L.T. (1995), Training Effectiveness Handbook, McGraw-Hill, New York.

Slovic, P., Fischhoff, B. and Lichtenstein, S. (1980), 'Facts and Fears: Understanding Perceived Risk', In Schwing, R.C. and Albers, W.A. (Eds.) Societal Risk Assessment, Plenum Press, New York, pp. 102-127.

't Hart, P. (1997), 'Preparing Policy Maker's for Crisis Management: The Role of Simulations', Journal of Contingencies and Crisis Management, 5, (4), 207-215.

Turner, B. and Pidgeon, N. (1997), Man-made Disasters, Second Edition, Butterworth Heinemann, Oxford.

United States National Response Team (1990), Developing a Hazardous Materials Exercise Program, U.S. Government Printing Office, Washington, D.C. 